\begin{tabular}{|l|l|}
\hline $\begin{array}{l}\text { Running title: Chen } \text { et al.: Host choice } \\
\text { similis }\end{array}$ & Guo-Hua Huang \\
& College of Plant Protection, \\
& Hunan Agricultural \\
& University, Changsha, Hunan 41 \\
& 0 128, China \\
& Tel.: +86-159-7411-2878 \\
& fax: +86-(0)731-8461-8163 \\
& Email: ghhuang@ $@$ \\
\hline
\end{tabular}

\title{
A place to grow? Host choice and larval performance of Microplitis similis in the host Spodoptera litura
}

Xu-Yang Chen ${ }^{1}$, R. J. Hopkins ${ }^{2}$, Yi-Pei Zhao ${ }^{1}$, Guo-Hua Huang ${ }^{{ }^{*}}$

1. Hunan Provincial Key Laboratory for Biology and Control of Plant Diseases and Insect Pests, Hunan Agricultural University, Nongda Road 1, Furong District,

Changsha, Hunan 410128, P. R. CHINA;

2. Faculty of Engineering and Science, University of Greenwich, Chatham Maritime, Kent, ME 4 4TB, United Kingdom

* Corresponding author: ghhuang@ hunau.edu (G.-H. Huang) 


\section{Abstract}

Host selection is a key stage in the lifecycle of parasitoids, and is critical to both their function in control and to the maintenance of their population. The solitary endoparasitoid Microplitis similis (Hymenoptera: Braconidae), is a potential biological control agent of Spodoptera litura larvae (Lepidoptera: Noctuidae). In this study, we examined the preference $M$. similis exhibits for different instars of the host, host instar effects on parasitoid development and the weight gain and food consumption of different instars of parasitized larvae. In no-choice tests, parasitization rates were highest in second and early third instar larvae, and no fourth or fifth instar hosts were parasitized. When provided with a choice of first to late third instars host larvae, $M$. similis preferred to parasitize early third instar host larvae $(41 \%)$ with a selection coefficient of 0.37 . All morphometric features of wasp offspring increased with increasing age of the host at parasitization. A lower proportion of females emerged from first instar larvae than any other instar. Parasitized S. litura larvae showed a pronounced reduction in food consumption and weight gain. Microplitis similis may have the potential to significantly suppress population growth and the damage caused by $S$. litura.

Keywords Microplitis similis, Spodoptera litura (Fabricius), Host age preference, Weight gain, Food consumption

\section{Introduction}

The oriental leafworm moth, Spodoptera litura (Fabricius) (Lepidoptera: Noctuidae) has a worldwide distribution, can migrate long distances, and has a high reproductive rate (Shad et al. 2012). Spodoptera litura is a polyphagous insect pest which has been 
recorded from over 100 families and causes extensive damage to tobacco, cotton, soybean and cruciferous vegetables (Tuan et al. 2014). Oriental leafworm was recently reported to cause economic losses of about $\$ 44.7$ million to soybean in the Kota region of Rajasthan (Dhaliwal et al. 2010). Conventional control of noctuid pests chiefly relies on chemical pesticides, and the frequent application of insecticide has led to the rapid evolution of insecticide resistance in the field (Ahmad et al. 2007; Su et al. 2012).

Biological control is an important component of successful Integrated Pest Management (IPM) programs. The use and enhancement of natural enemies of the insect pests plays an important role in IPM, and parasitoids are considered to be important biological control agents against Lepidopteran pests (Mills 2009; Barzman et al. 2015). He and Liu (2002) list hymenopterous parasitoids of S. litura from China, including 29 primary parasitoids and 11 hyperparasitoids. The genus Microplitis contains 200 species (Ernandez-Triana et al. 2015) and M. similis Lyle (Hymenoptera: Braconidae) is a solitary endoparasitoid, with hosts including Agrotis ypsilon (Rottemberg), Spodoptera exigua (Hübner) and S. litura (Fabricius) (Shepard and Barrion 1998; Ranjith et al. 2015). Microplitis similis was first recorded in India (Wilkinson 1930) and identified as a vector of Heliothis virescens ascovirus $3 \mathrm{~h}$ only recently ( $\mathrm{Li}$ et al. 2016).

Host species much influence the behavior of parasitoid wasps (Kapranas and Tena 2015) and their development within the host (Mawela et al. 2013). In addition, the instar of host larvae has received widespread attention, and often influences adult oviposition choice and the performance of immatures (Hu and Vinson 2000; Nussbaumer and Schopf 2000; Mironidis and Savopoulou-Soultani 2008). Once an egg has been laid in the host, many parasitoid larvae grow by feeding on silk glands 
and haemolymph (Jackson et al. 1978), resulting in a reduction of food intake and weight gain of host larvae when compared to the non-parasitized larvae (Mironidis and Savopoulou-Soultani 2009).

In this paper, we describe the host instar preference of $M$. similis, the effects of $S$. litura larval stage on the development of $M$. similis offspring and weight gain and food consumption of parasitized hosts. This study provides knowledge for assessing the biological control potential of $M$. similis against $S$. litura, and for finding the most suitable conditions for mass production of the parasitoid.

\section{Materials and Methods}

\section{$\underline{\text { Study insects }}$}

Spodopera litura larvae were reared on pinto bean-based diets until pre-pupation at $30 \pm 1{ }^{\circ} \mathrm{C}, 60 \pm 5 \% \mathrm{RH}$, with a photoperiod of 14: 10 (L: D) h (Burton 1970; Li et al. 2015). First to fifth S. litura instars were used for these experiments.

A colony of M. similis wasps was established in the laboratory in May 2013. Cocoons of $M$. similis were collected from wild second and third instar S. litura larvae in a vegetable field near Hunan Agricultural University, Changsha City, Hunan Province, China. The parasitoids were reared on $S$. litura larvae with a $40 \%$ honey solution as food at $30{ }^{\circ} \mathrm{C}$ ( $\mathrm{Li}$ et al. 2015). For this experiment, $M$. similis adults were cultured with $30 \%$ honey solution at $30 \pm 1{ }^{\circ} \mathrm{C}, 60 \pm 5 \% \mathrm{RH}$, with a photoperiod of 14: 10 (L: D). All experiments were conducted under the same environmental conditions described above. The species identification of $M$. similis was referenced to Li et al. (2015). Molecular identification based on the barcoding col sequence following Huang's methods (Huang et al. 2011) was performed based on the col sequence of $M$. similis (GenBank Access Number: KX077544) and the 
Microplitis col sequences in NCBI, the phylogenetic relationship of M. similis is shown in supplementary figures. There is a growing literature demonstrating that col will reliably discriminate a diverse range of taxa at the species level. Therefore, the identification of $M$. similis was also conducted on the basis of phylogeny using DNA barcoding.

\section{Instar Preferences}

Experimental studies were conducted on four larval stages (third instar larvae were split into early and late stages after He et al. (2014)) of S. litura using no-choice and choice tests in environmental chambers. In no-choice tests, twenty individual larvae of the same instar and one mated female wasp were kept in a plastic tube $(10 \mathrm{~cm}$ length $\times 3 \mathrm{~cm}$ diameter, $\mathrm{n}=30$ per treatment) containing $30 \%$ honey solution and a piece of sweet potato leaf. After $24 \mathrm{~h}$ exposure, the host larvae were placed in a plastic case $(4.5 \times 12.0 \times 7.0 \mathrm{~cm})$ and provided with the standard diet. The larvae were cultured in the environment chambers, and checked daily until they pupated or died. Host larvae that died before parasitoid cocooning or pupation were dissected, to ascertain whether they contained parasitoid eggs or larvae. The number of parasitoid cocoons was recorded. Successful parasitization was defined by a host larvae yielding at least one parasitoid, and parasitism was calculated as number of successful parasitized larvae/number of larvae tested $\times 100$.

In choice tests, high densities of fourth and fifth instar S. litura larvae exhibited cannibalistic behavior, which resulted in high mortality. Therefore, only first to late third instars larvae were provided for the choice test. Eighty hosts (20 of each stage) were exposed to 10 parasitoids in a plastic cage $(6 \times 18 \times 12 \mathrm{~cm})$. After $24 \mathrm{~h}$ exposure, the host larvae were separated and cultured in an environment chamber under the same conditions as described above for rearing. The host larvae were checked and 
recorded daily as described above. This experiment was replicated 5 times. Selection coefficient of M. similis was calculated as follows (Cock 1978; Chu et al. 2014).

Selection Coefficient $=R_{i} / \sum_{i=1}^{\mathrm{m}} R i$. Where $R_{i}=$ the percentage parasitism during host instars $i ; \mathrm{m}=$ number of host instars tested.

\section{Host instar effects on the development of $M$. similis}

First to late third instar S. litura were tested for their effect on the development and body size of $M$. similis. Adult parasitoid were taken from the culture as described in the no-choice tests. The development times from egg to cocoon, cocoon to adult, emergence percentage and sex ratio were recorded for each treatment. Adult parasitoids which emerged were then checked daily until the host died. Adult body length, front wing length and hind-tibia length of each parasitoid were measured under Motic Digital Microscope Mutual System (He et al. 2014).

\section{Food consumption and weight gain of parasitized hosts}

The first, second, early third and late third instar S. litura were exposed individually to two mated female wasps in a plastic tube (as above). Once each larvae received an oviposition, it was immediately removed. These parasitized host larvae were examined for the body weight and food consumption each day until death or pre-pupation. These data were compared with corresponding data from non-parasitized larvae, which were reared separately from the fifth day. A piece of sweet potato leaf was placed in a test tube without larvae to estimate the percentage of moisture lost daily from a leaf in the environment chambers. The parasitized/unparasitized larvae were weighed on an analytical balance (Mettler Toledo, China). The following formula was used to calculate the food consumption (Chu et al. 2014). 
Proportion of daily moisture loss $=\frac{\text { fresh weight after dehydrating }(\mathrm{mg})}{\text { initial fresh weight of leaf }(\mathrm{mg})}$;

Food consumption $(\mathrm{mg})=$ initial fresh weight of leaf $(\mathrm{mg}) \times$ Proportion of daily moisture loss - fresh weight after feeding (mg).

\section{Statistical analysis}

All data analyses were performed using SPSS 16.0 for Windows (SPSS Inc ${ }^{\circledR}$, MA, version 16.0), and checked for normality and homoscedasticity (Qiu et al. 2013). When the data did not fit a normal distribution, the parameters, such as host parasitism, emergence percentage and female rate were first transformed by $\arcsin x$, and the parameters, such as the developmental durations were first transformed by $\log _{10} x$. The body length and front wings length were first transformed by lngamma $x$. The Duncan's multiple range test was used as a one way-analysis of variance test ( $\alpha=0.05$ ) in comparing the means between treatments.

For weight gain and food consumption of parasitized hosts data, a two way-analysis of variance test ( $a=0.01$ ) was conducted to show the impact of both instar and level parasitism (parasitized and non-parasitized) on the change of weight gain and food consumption of parasitized hosts (McLoud 2011).

\section{$\underline{\text { Results }}$}

\section{Instar preference}

Microplitis similis did not parasitize fourth and fifth instars larvae, which possess strong defensive behavioral reactions. Parasitism in no choice experiments was highest in second and third instar, and lowest in first instar $(F=49.01 ; \mathrm{df}=3,117 ; P$

$<0.05$; Fig. 1). When provided with a choice of first to late third instars host larvae (Fig. 2), early third and second instars $S$. litura were parasitized at the highest rates, 
followed by first and late third instars $(F=7.43$; $\mathrm{df}=3,20 ; P<0.05)$. There was also significant variation in the selection coefficient among instars $(F=26.39$; $\mathrm{df}=3$, $20 ; P<0.05)$, being highest in the early third instar at and lowest in the late third instar (Fig. 3).

\section{Effects of $S$. litura stage on the development of $M$. similis offspring}

Egg-cocoon development of $M$. similis in first instar S. litura was $7.06 \pm 0.06$ days, and increased with successive instars to $8.10 \pm 0.08$ days in the late third instar of $S$. litura $(F=41.22 ; \mathrm{df}=3,504 ; P<0.05)$ (Table 1$)$. Cocoon-adult development in late third instar larvae was significantly longer than in other host instars $(F=38.91$; df $=3,504 ; P<0.05)$. It just took $4.13 \pm 0.06$ days for $M$. similis to develop from cocoon-adult in the first instar host. The development duration of the parasitoid from egg to adult emergence was longest in the late third instar larvae, followed by the early third instar, second instar and finally first instar $(F=110.78 ; \mathrm{df}=3,504 ; P<$ 0.05). The stage of the host parasitized had no effect on the percentage of parasitoids successfully emerging $(F=0.493 ; \mathrm{df}=3,93 ; P=0.688)$.

The female rate was lower for adults emerging from first instar larvae than from other host instars $(F=3.90 ; \mathrm{df}=3,35 ; P<0.05)$. The female rate did not differ between adults emerging from the older instars $(F=3.90 ; \mathrm{df}=3,35 ; P=0.248)$. In the aspect of morphometric features, parasitoid body length, fore wing length, and hind tibia length were affected by all host instars (Table 2). All the above morphometric features increased with the increase of host instar age at parasitism. All of the morphometric features (body length, fore wing length, hind tibia length), were greatest in late third instar larvae (body length: $F=102.33$; $\mathrm{df}=3,231 ; P<0.05$; 
fore wing length: $F=79.10 ; \mathrm{df}=3,231 ; P<0.05$; hind tibia length: $F=42.52$; $\mathrm{df}=$ $3,231 ; P<0.05)$..

\section{Weight gain and food consumption of parasitized hosts}

Parasitized $S$. litura showed a pronounced reduction in weight gain (ANOVA: level of parasitism, $F=77.30 ; \mathrm{df}=1,255 ; P<0.01$; host instars, $F=130.07$; df = 3, 255; $P<$ 0.01, interaction: $\mathrm{F}=543.40 ; \mathrm{df}=1,255 ; P<0.01$ ) (Fig. 4). All instar of $S$. litura larvae were affected, although this was most marked in the late third instar, which were the largest when unparasitized. The daily food consumption of parasitized hosts showed a clear reduction in the food consumed (ANOVA: level of parasitism, $F$ $=86.72 ; \mathrm{df}=1,256 ; P<0.01$; host instar, $F=91.92 ; \mathrm{df}=3,256 ; P<0.01$, interaction: $F=548.45$; df $=1,255 ; P<0.01$ ) (Fig. 5). All instar of $S$. litura larvae were affected, and this was most notable in the late third instar, which had the highest rate of food consumption when unparasitized. The maximum food consumption of the host after parasitism was dependent on host instar of $S$. litura into which the parasite had oviposited.

\section{$\underline{\text { Discussion }}$}

We found that female Microplitis similis were more likely to parasitize second and early third instars $S$. litura larvae in both choice and no-choice tests, and immature $M$. similis grew well in second and early third instars S. litura larvae. Microplitis similis had a lower success rate parasitizing late third instar larvae, most likely as an effect of the older instar larvae having a strong defensive behavior (Ameri et al. 2014). Early third instar hosts ranked higher than other instars in terms of suitability, yielding 
offspring of greater body size, although they required a slightly longer development time. Parasitized S. litura larvae exhibited a decreased rate of weight gain and daily food consumption in comparison to unparasitized larvae. In summary, M. similis females selected the best host stage for the development of their offspring, and had the beneficial effect of greatly reducing consumption by host larvae. Thus, it is apparent that enhancing $M$. similis can contribute as a component of an IPM strategy for S. litura in economic crops.

Host evaluation and selection by hymenopteran parasitoids is a key event, because high host suitability is critical to the growth and development of parasitoid larvae (Vinson 1990; Murillo et al. 2013). Under natural conditions, adult females accept or reject host larvae for oviposition, and the success rates of parasitism are low when inoculating small and large hosts due to early host death and parasitoid injury, respectively (Wei et al. 2014). In our study, we identified the preferred host instar range of $M$. similis to be first to late third instars $S$. litura larvae. When $M$. similis sought to parasitize the fourth and fifth instars larvae, the larvae demonstrated strong defensive behaviors. When the parasitoid used antennae to evaluate fourth and fifth instars larvae, the larvae would twist the head and attack the wasp, resulting in wounding and even death. Selectivity by parasitoids, in terms of host instar, has been demonstrated by He et al. (2014) who found that Euplectrus laphygmae (Ferrière) also could parasitize first to late third instars $S$. litura larvae, but parasitized second and third instar hosts at a higher rate in no-choice tests. However, E. laphygmae prefer to parasitize early third S. litura larvae when offered a choice of second, early third and late third.

In koinobionts, parasitoid larvae have two kinds of feeding strategies, tissue-feeding strategies and hemolymph-feeding (Harvey and Malcicka 2016). The 
vast majority of Microgastrinae parasitoid larvae consume most host tissues, including in the genera Microgaster, Apanteles and Dolichogenidea (Harvey and Malcicka 2016). The larva of Hyposoter ebeninus consumed the entire Pieris rapae larvae (Gauld and Bolton 1988). On the other hand, microgastrines in the genera Microplitis and Cotesia are hemolymph feeders (Malcicka and Harvey 2015). Different hosts provide quantitative and qualitative variation in nutrition, which consequently influence the growth and ultimately the morphometric features of parasitoid offspring from different host instars or host species (Harvey 2000; Harvey and Strand 2002; Mironidis and Savopoulou-Soultani 2009). In general, larger hosts yield more offspring (Harvey 2000; Stockermans and Hardy 2013) and female offspring often hatch from large high quality hosts and male offspring from low quality hosts (Charnov et al. 1981; Pekas et al. 2016). Strand et al. (1988) also reported that Microplitis demolitor achieved larger body size in the larger later instars of Heliothis virescens larvae, in a manner similar to that found here for $M$. similis developing in S. litura. The female $M$. similis laid few fertilized eggs in small early instar larvae. The low female ratio obtained from first instar Microplitis tuberculifer larvae (Chu et al. 2014) is similar also to this study.

Harvey et al. (2010) reported that the intensity of resourced-related constraints on parasitoid development also varies from one parasitoid species to another. In koinobiont parasitoids, the host represents a potentially dynamic resource, koinobiont parasitoids attack hosts that continue feeding and growing during immature parasitoid development (Harvey 2005; Harvey et al. 2010). In our study, larvae of S. litura parasitized by $M$. similis continue feeding and growing until the mature parasitoid larva emerged from the body of the host, and the parasitized herbivores showed greatly reduced weight gain and food consumption compared to unparasitized larvae, 
whatever the instar parasitized. This result is similar to larvae of Mythimna separata parasitized by $M$. mediator (Li et al. 2006). In terms of host regulation, early instar hosts provide insufficient nutrients to koinobiont parasitoids, so parasitoids do not immediately inhibit lepidopteran host growth until the host becomes larger. In contrast, koinobiont parasitoids attack later instar lepidopteran hosts greatly reducing host growth (Harvey et al. 1994, 2014). In this study, significant decreases in weight gain of the first instar S. litura larvae parasitized by M. similis began on the fifth day, whereas late third instar host larvae showed a significant decrease in weight gain only two days after being parasitized by $M$. similis. Very similar results were obtained for Hyposoter didymator parasitizing Helicoverpa armigera (Mironidis and Savopoulou-Soultani 2008). Changes in the food consumption of S. litura parasitized by $M$. similis began on different days after parasitism in different host instars, a result similar to that found for M. tuberculifer on S. exigua (Chu et al. 2014). The influence of a parasitoid on host feeding is of key importance to the role of parasitoids in IPM strategies, and from a plant protection perspective it is clearly an advantage if parasitization results in a larvae reducing or halting feeding. In that $M$. similis results in a progressive reduction in feeding over a moderate period, it is less favorable than a species which rapidly causes the host to stop feeding.

We found that $M$. similis parasitized second and early third instar larvae at a higher rate than other larvae, and this was optimal for the parasitoid larvae to develop and survive. Most parasitoids can successfully complete their life cycle in different instar host larvae, but with different performance in different instars. This is important for IPM of the pest, but also is important information for mass-rearing of M. similis. In the current study, we found the most suitable host stage for the growth of $M$. similis to be early third instar. However, environmental factors, such as temperature, humidity 
and photoperiod, as well as the host species, body size, and instar would influence parasitoid's mass production. The details to optimize rearing of $M$. similis will require further study, but the control of S. litura with parasitic natural enemies can potentially provide a component of an overall IPM strategy.

\section{Acknowledgments}

The authors thank Miss Shun-Ji Li, Miss Zhi Xu, Mr. Yun-Xuan Zhang, Mr. Xi-Ju Xia for her kind help in the experiments. This study was supported by Natural Science Foundation of China (31371995), the Hunan Provincial Natural Science Foundation for Distinguished Young Scholar of China (14JJ1023) and Hunan Collegiate Technology Innovation Project (SCX1411). This article was much improved by the precise comments of Jeff Harvey and a second anonymous reviewer.

\section{$\underline{\text { References }}$}

Ahmad, M., Arif, M. I., and Ahmad, M. 2007. Occurrence of insecticide resistance in field populations of Spodoptera litura (Lepidoptera: Noctuidae) in Pakistan. Crop Prot. 26: 809-817.

Ameri, M., Rasekh, A., and Michaud, J. P. 2014. Body size affects host defensive behavior and progeny fitness in a parasitoid wasp, Lysiphlebus fabarum. Entomol Exp Appl. 150: 259-268.

Barzman, M., Bàrberi, P., Birch, A. N. E., Boonekamp, P., Dachbrodt-Saaydeh, S., Graf, B., and Lamichhane, J. R. 2015. Eight principles of integrated pest 
management. Agron Sustain Dev. 35: 1199-1215.

Burton, R. L. 1970. A low-cost artificial diet for the corn earworm. J Econ Entomol. 63: 1969-1970.

Charnov, E., Los-den Hartogh, R. L., Jones, W. T., and Van den Assem, J. 1981. Sex ratio evolution in a variable environment. Nature. 289: 27-33.

Chu, Y. N., Michaud, J. P., Zhang, J., Lia, Z., Wang, Y. Z., Chen, H., Li, J. C., Lu, Z. Y., Zhang, Q. W., and Liu, X. X. 2014. Performance of Microplitis tuberculifer (Hymenoptera: Braconidae) parasitizing Mythimna separata (Lepidoptera: Noctuidae) in different larval instars. Biol Control. 69: 18-23.

Cock, M. J. W. 1978. The assessment of preference. J Anim Ecol. 47: 805-816.

Dhaliwal, G. S., Jindal, V., and Dhawan, A. K. 2010. Insect pest problems and crop losses: changing trends. Indian J Ecol. 37: 1-7.

Ernandez-Triana, J. L., Whitfield, J. B., Smith, M. A., Kula, R., Hallwachs, W., and Janzen, D. 2015. Revision of the genera Microplitis and Snellenius (Hymenoptera, Braconidae, Microgastrinae) from Area de Conservacion Guanacaste, Costa Rica, with a key to all species previously described from Mesoamerica. Deut Entomol Z, 62: 137-201.

Gauld, I., and Bolton, B. 1988. The Hymenoptera. Oxford University Press, Oxford, UK.

Harvey, J. A. 2000. Dynamic effects of parasitism by an endoparasitoid wasp on the development of two host species: implications for host quality and parasitoid fitness. Ecol Entomol. 25: 267-278.

Harvey, J. A. 2005. Factors affecting the evolution of development strategies in parasitoid wasps: the importance of functional constraints and incorporating complexity. Entomol Exp Appl. 117: 1-13. 
Harvey, J. A., Harvey, I. F., and Thompson, D. J. 1994. Flexible larval growth allows use of a range of host sizes by a parasitoid wasp. Ecology 75: 1420-1428.

Harvey, J. A., and Malcicka, M. 2016. Nutritional integration between insect hosts and koinobiont parasitoids in an evolutionary framework. Entomol Exp Appl, 159: 181-188.

Harvey, J.A., Sano, T., and Tanaka, T. 2010. Differential host growth regulation by the solitary endoparasitoid, Meteorus pulchricornis in two hosts of greatly differing mass. J Insect Physiol. 56:1178-1183.

Harvey, J. A., and Strand, M. R. 2002. The developmental strategies of endoparasitoid wasps vary with host feeding ecology. Ecology. 83: 2439-2451.

Harvey, J. A., Visser, B., Le Lann, C., De Boer, J., Ellers, J., and Gols, R. 2014. Convergence and divergence in direct and indirect life-history traits of closely related parasitoids (Braconidae: Microgastrinae). Evol Biol, 41: 134-144.

He, J. Y., Chen, Z. S., Chen, X. Y., Zhang, Y. X., and Huang, G. H. 2014. Biological characteristics of Euplectrus laphygmae Ferrière (Hymenoptera: Eulophidae). Chin J Biol Contr. 30: 453-459.

He, J. H., and Liu, Y. Q. 2002. List of hymenopterous parasitoid of Spodoptera litura Fabricius from China. Nat Enem Insect. 24: 128-137.

Huang, G. H., Chen, L. S., Hirowatari, T., Nasu, Y., and Wang, M. 2011. A revision of the Monopis monachella species complex (Lepidoptera: Tineidae) from China. Zool J Linn Soc-Lond. 163: 1-14.

Hu, J. S., and Vinson, S. B. 2000. Interaction between the larval endoparasitoid Campoletis sonorensis (Hymenoptera: Ichneumonidae) and its host the tobacco budworm (Lepidoptera: Noctuidae). Ann Entomol Soc Am. 93: 220-224.

Jackson, C. G., Delph, J. S., and Neemann, E. G. 1978. Development, longevity 
and fecundity of Chelonus blackburni [Hym.: Braconidae] as a parasite of Pectinophora gossypiella [Lep.: Gelechiidae]. Entomophaga. 23: 35-42.

Kapranas, A., and Tena, A. 2015. Encyrtid parasitoids of soft scale insects: Biology, behavior, and their use in biological control. Annu Rev Entomol. 60: 195-211.

Li, J., Coudron, T. A., Pan, W., Liu, X., Lu, Z., and Zhang, Q. 2006. Host age preference of Microplitis mediator (Hymenoptera: Braconidae), an endoparasitoid of Mythimna separata (Lepidoptera: Noctuidae). Biol Control. 39: $257-261$.

Li, S. J., Hopkins, R. J., Zhao, Y. P., Zhang, Y. X., Hu, J., Chen, X. Y., Xu, Z., and Huang, G. H. 2016. Imperfection works: Survival, transmission and persistence in the system of Heliothis virescens ascovirus 3h (HvAV-3h), Microplitis similis and Spodoptera exigua. Sci Rep. 6: 21296.

Li, S. J., Huang, J. P., Chang, Y. Y., Quan, S. Y., Yi, W. T., Chen, Z. S., Liu, S. Q., Cheng, X. W., and Huang, G. H. 2015. Development of Microplitis similis (Hymenoptera: Braconidae) on two candidate host species, Spodoptera litura and Spodoptera exigua (Lepidoptera: Noctuidae). Fla Entomol. 98: 736-741.

Malcicka, M., and Harvey, J.A. 2015. Development of two related endoparasitoids in larvae of the diamondback moth, Plutella xylostella (Lepidoptera: Plutellidae). BioControl 60: 149-155.

Mawela, K. V., Kfir, R., and Krüger, K. 2013. Effect of temperature and host species on parasitism, development time and sex ratio of the egg parasitoid Trichogrammatoidea lutea Girault (Hymenoptera: Trichogrammatidae). Bio Control. 64: 211-216.

McLoud, L. A. 2011. Microplitis croceipes (Hymenoptera: Bracondiae): A Life History Study and In Vitro Rearing. Texas A \& M University, College Station, 
Texas, USA. 75 pp.

Mills, N. 2009. "Egg parasitoids in biological control and integrated pest management." Egg parasitoids in agroecosystems with emphasis on Trichogramma. Springer Netherlands pp. 389-411.

Mironidis, G. K., and Savopoulou-Soultani, M. 2008. Development, survivorship, and reproduction of Helicoverpa armigera (Lepidoptera: Noctuidae) under constant and alternating temperatures. Environ Entomol. 37: 16-28.

Mironidis, G. K., and Savopoulou-Soultani, M. 2009. Development, survival and growth rate of the Hyposoter didymator-Helicoverpa armigera parasitoid-host system: effect of host instar at parasitism. Biol Control. 49: 58-67.

Murillo, H., Hunt, D.W.A., and VanLaerhoven, S.L. 2013. Host suitability and fitness-related parameters of Campoletis sonorensis (Hymenoptera: Ichneumonidae) as a parasitoid of the cabbage looper, Trichoplusia ni (Lepidoptera: Noctuidae). Biol Control. 64: 10-15.

Nussbaumer, C., and Schopf, A. 2000. Development of the solitary larval endoparasitoid Glyptapanteles porthetriae (Hymenoptera: Braconidae) in its host Lymantria dispar (Lepidoptera: Lymantriidae). Eur J Entomol. 97: 355-362.

Pekas, A., Tena, A., Harvey, J.A., Garcia-Marí, F., and Frago, E. 2016. Host size and spatiotemporal patterns mediate the coexistence of specialist parasitoids. Ecology. 97: 1345-1356.

Qiu, B., Zhou, Z., and Xu, Z. 2013. Age preference and fitness of Microplitis manilae (Hymenoptera: Braconidae) reared on Spodoptera exigua (Lepidoptera: Noctuidae). Fla Entomol. 96: 602-609.

Ranjith, A. P., Rajesh, K. M., and Nasser, M. 2015. Taxonomic studies on oriental Microplitis Foerster (Hymenoptera: Braconidae, Microgastrinae) with 
description of two new species from south India. Zootaxa. 3963: 369-415.

Shad, S. A., Sayyed, A. H., Fazal, S., Saleem, M. A., Zaka, S. M., and Ali, M. 2012. Field evolved resistance to carbamates, organophosphates, pyrethroids, and new chemistry insecticides in Spodoptera litura Fab. (Lepidoptera: Noctuidae). J Pest Sci. 85: 153-162.

Shepard. B. M., and Barrion, A. T. 1998. Parasitoids of insects associated with soybean and vegetable crops in Indonesia. J Agric Entomol. 15: 239-272.

Stockermans, B. C., and Hardy, I. C. W. 2013. Subjective and objective components of resource value additively increase aggression in parasitoid contests. Biol Letters. 9: 20130391.

Strand, M.R., Johnson, J.A., Culin, J.D. 1988. Developmental interactions between the parasitoid Microplitis demolitor (Hymenoptera: Braconidae) and its host Heliothis virescens (Lepidoptera: Noctuidae). Ann Entomol Soc Am. 81: $822-830$.

Su, J., Lai, T., and Li, J. 2012. Susceptibility of field populations of Spodoptera litura (Fabricius) (Lepidoptera: Noctuidae) in China to chlorantraniliprole and the activities of detoxification enzymes. Crop Prot. 42: 217-222.

Tuan, S. J., Lee, C. C., and Chi, H. 2014. Population and damage projection of Spodoptera litura (F.) on peanuts Arachis hypogaea (L.) under different conditions using the age-stage, two-sex life table. Pest Manag Sci. 70: 805-813.

Vinson, S. B. 1990. Physiological interactions between the host genus Heliothis and its guild of parasitoids. Arch Insect Biochem. 13: 63-81.

Wei, K., Tang, Y. L., Wang, X. Y., Cao, L. M., and Yang, Z. Q. 2014. The developmental strategies and related profitability of an idiobiont ectoparasitoid Sclerodermus pupariae vary with host size. Ecol Entomol. 39: 101-108. 
Wilkinson, D. 1930. A revision of the Indo-Australian species of the genus Microplitis (Hymenoptera: Braconidae). B Entomol Res. 21: 23-27. 


\section{Tables}

Table 1. Mean $( \pm \mathrm{SE})$ developmental time, percentage of emergence and female rate of $M$. similis in different developmental stage of $S$. litura

Table 2. The effect of host stages on body size of M. similis (M $\pm \mathrm{SE})$

\section{Figure legends}

Figure 1. Mean $( \pm$ SE) parasitism of $M$. similis parasitizing $S$. litura in no-choice tests. Note: The $\mathrm{x}$ axis values represent 1 = first instar larvae, $2=$ second instar larvae, $3 \mathrm{E}=$ early third instar larvae, $3 \mathrm{~L}=$ late third instar larvae, respectively. The same letters above the same bars represent no significant differences in parasitism of the different host instars (Duncan test, $P<0.05$ )

Figure 2. Mean (+ SE) parasitism of $S$. litura larvae of various instars when parasitized by $M$. similis in choice tests. Note: The $\mathrm{x}$ axis values represent $1=$ first instar larvae, $2=$ second instar larvae, $3 \mathrm{E}=$ early third instar larvae, $3 \mathrm{~L}=$ late third instar larvae, respectively. The same letters above the same bars represent no significant differences in parasitism of the different host instars (Duncan test, $P<0.05$ )

Figure 3. Selection coefficient of S. litura larvae of various instars when parasitized by M. similis in choice tests. Note: The $\mathrm{x}$ axis values represent $1=$ first instar larvae, $2=$ second instar larvae, $3 \mathrm{E}=$ early third instar larvae, $3 \mathrm{~L}=$ late third instar larvae, respectively. The same letters above the bars represent no significant differences in selection coefficient of the different host instars

(Duncan test, $P<0.05$ )

Figure 4. Weight gain by hosts parasitized in different larval instars and un-parasitized hosts. (a) Weight of parasitized and un-parasitized first instar S. exigua, (b) Weight of parasitized and non-parasitized second instar S. exigua, (c) Weight of parasitized and non-parasitized early third instar S. exigua, and (d) Weight of parasitized and non-parasitized late third instar S. exigua, Note: 
1-d-old S. litura larvae were not parasitized, so they were used at 2 days old. Control:

Un-parasitized larvae. Weight gain of parasitized / non-parasitized 1-d-old to 4-d-old larvae was measured together, therefore the larvae (1-d-old to 4-d-old) values are mean, and the other values are mean $+\mathrm{SE}$.

Figure 5. Daily food consumption parasitized in different larval instars and un-parasitized hosts.

(a) Daily food consumption of parasitized and non-parasitized first instar S. exigua, (b) Daily food consumption of parasitized and non-parasitized second instar S. exigua, (c) Daily food consumption of parasitized and non-parasitized early third instar S. exigua, and (d) Daily food consumption of parasitized and non-parasitized late third instar S. exigua, Note: 1-d-old S. litura larvae were not parasitized, so they were used at 2 days old. Control $=$ non-parasitized larvae. Daily food consumption of parasitized / non-parasitized 1-d-old to 4-d-old larvae was measured together, therefore the larvae (1-d-old to 4-d-old) values are mean, and the other values are mean + SE. 